\title{
VIDA MÉDIA DE LABOR DOS ENFERMEIROS EGRESSOS DA ESCOLA DE ENFERMAGEM DE RIBEIRÃO PRETO, USP - BRASIL*
}

\author{
Geraldo Garcia Duarte** \\ Emilia Luigia Saporiti Angerami*** \\ Daisy Leslie Steagall Gomes**** \\ Iranilde José Messias Mendes****
}

DUARTE, G.G.; ANGERAMI, E.L.S.; GOMES, D.L.S.; MENDES, I.J.M. Vida média de labor dos enfermeiros egressos da Escola de Enfermagem de Ribeirão Preto, USP - Brasil. Rev.latino-am.enfermagem, Ribeirão Preto, v. 8 , n. 6 , p. 91-95, dezembro 2000.

O objetivo deste estudo é o de medir o tempo médio de trabalho em saúde dos egressos da Escola de Enfermagem de Ribeirão Preto da Universidade de São Paulo no periodo de 1957 a 1990, totalizando 1112 diplomados. Trata-se de um estudo descritivo de corte transversal, sendo que os dados foram coletados no periodo de março de 1992 a fevereiro de 1993. Medindo o tempo de trabalho em saúde, usamos a técnica denominada tábuas de sobrevivência de uso comum nos estudos de seguimento. A partir de nossos dados podemos estimar que a vida média de labor vale por 18,12 anos.

UNITERMOS: recursos humanos de enfermagem, trabalho, prática profissional

\section{INTRODUÇÃO}

A acelerada dinâmica de nossa realidade produz mudanças rápidas e simultâneas no ambiente social, econômico, nas estruturas familiares, demográficas e mutações, no mundo do trabalho. Mudam também as estruturas sanitárias, nem sempre para melhor. Como aproveitar estas mudanças para reduzir as desigualdades e promover melhor qualidade de assistência?

É fundamental que se ressalte que "não se constituirá um sistema de saúde superior ao atual sem uma política de recursos humanos séria e competente" (VIANNA, 1986, p.5) onde os novos atores que direta ou indiretamente influem na saúde participem ativamente na formulação de políticas e programas inerentes a nova estrutura organizacional e funcional que ora vai se constituindo.

Desta forma, faz-se necessária uma reorientação das politicas de recursos humanos, seja nos aspectos relativos à força de trabalho, seja naqueles vinculados à preparação de pessoal de saúde (VIANNA, 1986, p.6).

Tais considerações são de extrema importância no sentido de situar a problemática vivenciada no setor saúde, no que toca a sua reorientação e a questão também dos recursos humanos de enfermagem, que efetivamente constituem cerca de $50 \%$ do pessoal de saúde engajado no atendimento à população.

Dentro dos trabalhadores de enfermagem, os enfermeiros constituem $8,5 \%$ da força de trabalho. Constitui-se sem dúvida uma parcela relativamente pequena, se tomarmos a evidência que $63,8 \%$ do contigente da Enfermagem é formada por pessoal sem qualificação específica, os chamados atendentes (BRASIL, 1985).

O levantamento de Recursos e Necessidades em Enfermagem (ASSOCIAÇÃO BRASILEIRA DE ENFERMAGEM, 1980) reconheceu, pela primeira vez,

\footnotetext{
* Trabalho subvencionado pelo CNPq

** Professor Associado da Faculdade de Medicina de Ribeirão Preto da Universidade de São Paulo (Aposentado) e Membro do Grupo de Investigação em Recursos Humanos - Enfermagem da Escola de Enfermagem de Ribeirão Preto da Universidade de São Paulo - Brasil

*** Professor Titular do Departamento de Enfermagem Geral e Especializada e Membro do Grupo de Investigação em Recursos Humanos - Enfermagem da Escola de Enfermagem de Ribeirão Preto da Universidade de São Paulo. Endereço: Av. Bandeirantes, 3900 - Monte Alegre - 14040-902 - Ribeirão Preto - São Paulo - Brasil.

**** Professor Associado do Departamento de Enfermagem Materno-Infantil e Saúde Pública e Membro do Grupo de Investigação em Recursos Humanos - Enfermagem da Escola de Enfermagem de Ribeirão Preto da Universidade de São Paulo
} 
as dificuldades enfrentadas pelos profissionais quanto às suas condições de trabalho, rotatividade, alta evasão da profissão, insatisfação e falta de motivação, a baixa demanda para os cursos de graduação, evasão escolar e dificuldades no processo de formação profissional. Estes problemas, de forma talvez até mais intensa, se fazem presentes até os nossos dias. São muitas as dificuldades, e às vezes estas são percebidas como uma crise da profissão.

Abandonando o enfoque apenas profissional, e buscando a referência no movimento sanitário atual, e na constituição da força e do mercado de trabalho como processos dinâmicos, consideramos que as dificuldades identificadas carecem de ser estudadas. Essa realidade nos leva a repensar o papel social desempenhado pelo enfermeiro na sociedade brasileira atual, e dentro do setor saúde especificamente.

Muitas situações ligadas a recursos humanos em enfermagem, tem sido identificadas como necessárias e merecedoras de uma pesquisa cuidadosa, entre elas a crônica escassez de profissionais para atender à demanda crescente do mercado de trabalho.

TAYLOR (1976), em seus estudos, concluiu que a participação de enfermeiro na força de trabalho, sofre gradativamente, nos dez anos seguintes à sua graduação, um esvaziamento crescente, atingindo a uma repressão de $60 \%$ da totalidade da força de trabalho inicial. Com o distanciamento do período de graduação, $30 \%$ do total do grupo de enfermeiros estudados retornam ao processo produtivo, alcançando, em torno do vigésimo ano de graduação, aproximadamente, $70 \%$ da totalidade de profissionais que ingressaram na força de trabalho. A partir dessa data, eles se retiram paulatinamente até o afastamento definitivo.

MENDES (1982), realizou uma pesquisa, na Região Metropolitana de Belo Horizonte, constatando situação semelhante, indicando que $66,0 \%$ dos enfermeiros tinham menos de 10 anos de formados, observando brusca redução de enfermeiros no trabalho, à proporção que se distancia o tempo de graduação. Identificou que a participação do enfermeiro no mercado de trabalho, a partir de seu ingresso é de 10,17 anos como força de trabalho economicamente ativa.

ESTRYN-BEHAR \& POINSIGNON (1989) dizem que a maior parte dos enfermeiros interrompem suas atividades para cuidar dos filhos, ou para exercer outra profissão menos opressiva. Só as mais determinadas, as solteiras, continuam na carreira profissional. A ancianidade média das diplomadas, apesar de tudo, passou de 8 anos e meio em 1975 para 10 anos no início de 1986.

Na Itália, segundo CAVIOCHIOLI et al.(19961997, p.19) "o período de serviço do pessoal de enfermagem é reduzido (cerca de 10 anos), resultando um elevado turn-over".

O problema central que se configura pode ser descrito como uma acentuada escassez de enfermeiros, tanto no nível nacional como internacional. O fenômeno é descrito como resultante: da baixa procura aos cursos de enfermagem; aumento significativo da demanda aos serviços de saúde; condição de trabalho do enfermeiro, considerada por alguns autores como péssima. No Brasil são escassos os estudos relativos às condições de trabalho e permanência ou abandono do mercado de trabalho.

A resposta a estes problemas é de grande relevância, considerando-se investimento que se faz na formação de profissional, e a necessidade crescente de recursos humanos qualificados nos serviços.

Destaca-se também a importância de desenvolver modelos metodológicos, que permitam a realização de estudos com abordagens distintas das realizadas até o momento.

O objetivo deste estudo é o de tentar medir a vida média de labor em saúde dos egressos, do período de 1957 a 1990, da Escola de Enfermagem de Ribeirão Preto da Universidade de São Paulo (EERP-USP).

\section{MATERIAL E MÉTODO}

Trata-se de um estudo descritivo de corte transversal, cujos dados foram coletados no período de março de 1992 a fevereiro de 1993.

O universo deste estudo expressa o total dos alunos diplomados pela Escola de Enfermagem de Ribeirão Preto da Universidade de São Paulo (EERPUSP), no período de mencionado, totalizando 1112 diplomados.

A inexistência de um Banco de Dados na EERPUSP informando sobre a vida profissional de seus exalunos, resultou na busca de outras fontes como o Conselho Federal de Enfermagem (COFEN), Conselho Regional de Enfermagem do Estado de São Paulo (COREN-SP), Associação Brasileira de Enfermagem (ABEn), encontros pessoais, congressos, atividades sociais, locais de trabalho e outros.

Obtidos os dados solicitados, remeteu-se correspondência particular, contendo questionário relativo à busca de informações. Acrescenta-se que alguns graduados foram entrevistados pessoalmente, ou via telefônica.

O questionário compreendia uma orientação sobre a pesquisa e a forma de preenchimento. Três percursos eram possíveis:

a) destinado àqueles que não exerceram a profissão;

b) destinado àqueles que exerceram temporariamente a profissão;

c) destinado àqueles em exercício e aos aposentados.

Os dados informaram: 
- motivo de escolha da profissão e razões de não ingresso no mercado;

- tempo para ingresso no mercado de trabalho;

- razões de abandono da profissão, tempo de trabalho, áreas de maior permanência e desejo de retorno;

- justificativas para permanência na profissão e informações referentes às condições de trabalho.

\section{RESULTADOS E CONCLUSÃO}

A Escola de Enfermagem de Ribeirão Preto da
Universidade de São Paulo formou, no período de 1957 a 1990, 1112 enfermeiros. A porcentagem dos profissionais localizados compreendeu 91,46\% (1017), lembrando que destes $72,66 \%$ (808) respondeu ao questionário; ademais, registrou-se $0,99 \%$ (11) falecimentos.

Concernente a situação desses profissionais no mercado de trabalho, a maioria $81,81 \%$ (661) exercia atividades. Atingiu a aposentadoria 3,22\% (26) e desses $23,08 \%$ (6) retornaram ao trabalho motivados por razões salariais. Permaneceu em atividade por um determinado período de tempo $12,62 \%$ (102); enquanto 2,35\% (19) não chegou a exercer a profissão (Fig.1).
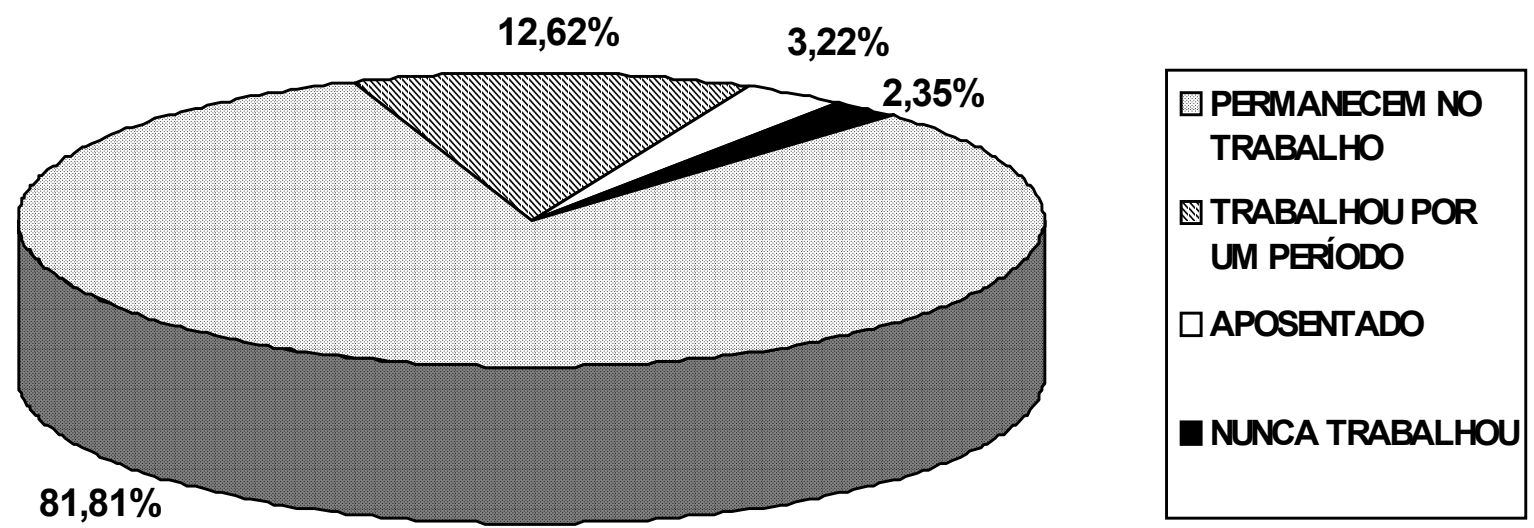

Figura 1 - Distribuição dos enfermeiros formados pela EERP-USP, segundo situação no mercado de trabalho

Tentando medir a "vida média de labor" dos egressos da EERP-USP, usamos a técnica denominada tábuas de sobrevivência de uso comum nos estudos de seguimento. Para tanto, a cada entrevistado foi associado um tempo de trabalho que foi medido da seguinte forma: a) O tempo zero é o da inserção no mercado, isto é, o instante em que recebe a "ordem legal" de exercer sua profissão.

b) O instante final é aquele em que o indivíduo se afasta definitivamente do serviço.

c) Como nosso sistema de coleta de dados é o de um corte num instante dado; encontramos na data da coleta de dados muitos indivíduos ainda em atividade e, nesses casos, diremos que houve uma "censura" no tempo de trabalho e os tempos desses entrevistados serão denominados censurados.

Os valores da variável tempo $\mathrm{T}$ podem ser agrupados em intervalos de classes definidos segundo nosso interesse $T_{1}, T_{2}, \ldots, T_{n}$.

A cada um desses intervalos podemos associar uma série de conceitos importantes para a análise de nossa variável, a saber:

a) Tamanho efetivo da amostra: que é definido pelo número de indivíduos que ingressaram no intervalo, subtraído pela metade do número de censurados durante o intervalo tentando dessa forma corrigir o número daqueles que estarão expostos ao risco de afastamento.

b) Probalidade de afastamento durante o intervalo, que é estimada pela proporção dos afastados dentre aqueles que constituem o tamanho efetivo da amostra $\hat{\mathrm{q}}$.

c) Probalidade de permanência estimada por $\hat{p}=1-\hat{q}$.

d) Função de sobrevivência $S(t)$ definida pela probabilidade de sobreviver ao tempo $t$,

$$
S(t)=P(T>t)
$$

$$
\begin{array}{ll}
\text { É clara que para a variável } S(t): & S(0)=1 \\
& S(00)=0
\end{array}
$$

Usando o conceito definido de probabilidade de permanência no $\mathrm{k}^{\mathrm{mo}}$ intervalo, $\mathrm{P}_{\mathrm{i}}$ teremos

$$
\mathrm{S}(\mathrm{k})=\mathrm{P}_{1} \times \mathrm{P}_{2} \times \ldots \times \mathrm{P}_{\mathrm{k}}
$$

que pode ser estimada por:

$$
\hat{S}(k)=\underset{i=1}{k} \hat{\mathbb{P}}_{\mathrm{i}}
$$

Usando as definições aqui propostas podemos construir a seguinte tabela de informações para nossos dados: 
Tabela 1 - Período de afastamento entre permanência no trabalho dos enfermeiros da Escola de Enfermagem de Ribeirão Preto formados de 1957 a 1990

\begin{tabular}{c|c|c|c|c|c|c}
\hline $\begin{array}{c}\text { PERIODO } \\
\text { (meses) }\end{array}$ & AFASTAMENTOS & CENSURAD O & $\begin{array}{c}\text { TAMANHO } \\
\text { EFETIVO }\end{array}$ & $\begin{array}{c}\text { PROP ORÇ̃̃o } \\
\text { AFASTAMENTO }\end{array}$ & $\begin{array}{c}\text { PROP ORÇÃo } \\
\text { PERMANÊNCLA }\end{array}$ & S OBREVIVENCIA \\
\hline $0 \mid--12$ & 27 & 0 & 808,0 & 0,0334 & 0,9666 & 1 \\
$12 \mid--72$ & 145 & 53 & 754,5 & 0,1922 & 0,8078 & 0,9666 \\
$72 \mid--132$ & 154 & 79 & 543,5 & 0,2833 & 0,7167 & 0,7808 \\
$132 \mid--192$ & 82 & 90 & 305,0 & 0,2688 & 0,7312 & 0,5596 \\
$192 \mid--252$ & 30 & 58 & 149,0 & 0,2013 & 0,7987 & 0,4091 \\
$252 \mid--312$ & 18 & 26 & 77,0 & 0,2338 & 0,7662 & 0,3268 \\
$312 \mid--372$ & 19 & 13 & 39,5 & 0,4810 & 0,5191 & 0,2503 \\
$372 \mid--432$ & 6 & 8 & 10 & 0,6000 & 0,4000 & 0,1299 \\
\hline
\end{tabular}

A partir de nossos dados podemos calcular a "vida média de labor" que é definida por

$$
\hat{\mathrm{u}}=1 \times \mathrm{t}_{1}+\mathrm{I}_{2} \mathrm{~S}\left(\mathrm{t}_{1}\right)+\mathrm{I}_{3} \mathrm{~S}\left(\mathrm{t}_{2}\right)+\ldots+\mathrm{I}_{\mathrm{n}} \mathrm{S}\left(\mathrm{t}_{\mathrm{n}-1}\right)
$$

e em nosso caso vale 18, 12 anos.

Queremos ressaltar que nossas estimativas são super estimadas gerados principalmente pela natureza de nossos dados e pela alta porcentagem de censurados (40,5\%), que por sua vez é influenciada pelo nosso sistema de coleta de informações, pois ele favoreceu de forma significativa os indivíduos ainda trabalhando.

\section{AVERAGE WORKING LIFE OF NURSES GRADUATING FROM THE UNIVERSITY OF SÃO PAULO AT RIBEIRÃO PRETO COLLEGE OF NURSING, BRAZIL}

This study aimed at measuring the average working time in health care of nurses graduating from the University of São Paulo at Ribeirão Preto College of Nursing, Brazil. This research includes 1,112 nurses who graduated from 1957 to 1990. It is a descriptive cross-sectional study. Data were obtained from March, 1992 to February, 1993. Statistical methods for survival data analysis were used. The average time was 18.12 years.

KEY WORDS: nursing human resources, job, professional practice

\section{VIDA MÉDIA PRODUCTIVA DE LOS ENFERMEROS EGRESADOS DE LA ESCUELA DE ENFERMERÍA DE RIBEIRÃO PRETO, UNIVERSIDADE DE SÃO PAULO, BRASIL}

El objetivo de este estudio es el de medir el tiempo medio de trabajo en salud de los egresados de la Escuela de Enfermería de Ribeirão Preto de la Universidad de São Paulo en el periodo de 1957 a 1990, totalizando 1112 egresados. Se trata de un estudio descriptivo de corte transversal, siendo que los datos fueron recolectados en el periodo de marzo de 1992 a febrero de 1993. Midiendo el tiempo de trabajo en salud, usamos la técnica denominada tablas de sobrevivencia de uso común en los estudios de seguimiento. A partir de nuestros datos podemos estimar que la vida media productiva está en 18,12 años.

TÉRMINOS CLAVES: recursos humanos de enfermería, trabajo, práctica profesional

\section{REFERÊNCIAS BIBLIOGRÁFICAS}

\section{ASSOCIAÇÃO BRASILEIRA DE ENFERMAGEM.}

Levantamento de recursos e necessidades em Enfermagem no Brasil - 1956/1958. Brasília, DF, 1980. $401 \mathrm{p}$.

02. BRASIL. Conselho Federal de Enfermagem. O exercício da Enfermagem nas Instituições de Saúde do Brasil:1982/1983. Rio de Janeiro: Conselho Federal de Enfermagem/Associação Brasileira de Enfermagem, 1985.
03. CAVIOCHIOLI, A. et al. Profissionalitá infermieristica: elementi essenziali, strumenti operativi. In: ANSELMI, M.L. II quadro de riferimento per la formulaziena del Budget del Personale Infermieristico nelle aziende sanitarie. Milano: University of Birgman/ Universitá Commerciale "L. Bocconi” Scuola direzione aziendale, 1996-1997. p. 19.

04. ESTRYN-BEHAR, M.; POINSIGNON, M. Travailler à l'hôpital. Paris: Berger - Levrault, 1989. 
05. MENDES, D.C. Recursos humanos em enfermagem.

Adequação da formação à utilização. In: CONGRESSO BRASILEIRO DE ENFERMAGEM, 34. Porto Alegre, 1982. Anais. Porto Alegre: ABEn, 1982. p.78-84.
06. TAYLOR Health manpower. In: TURKEY: an international research case study. Baltimore: Jonhs Hopkins Press, 1976.

07. VIANNA, Solon M. Saúde: descentralização e desburocratização. Brasília, DF, Setembro 1986. / mimeografado/. 